\title{
Zeichen setzen im digitalen Schreiben
}

\author{
Dürscheid, Christa
}

\begin{abstract}
Der vorliegende Beitrag befasst sich mit dem Schreiben an der Tastatur (digital) und nicht von Hand (analog). Hier aber stellt sich schon die erste Frage: Es ist ja auch möglich, mit einem Stift oder mit dem Finger auf einen Touchscreen zu schreiben. Handelt es sich dabei um ein analoges Schreiben (da von Hand) oder um ein digitales (da die derart eingegebenen Schriftzeichen vom Computer digital verarbeitet werden)?
\end{abstract}

DOI: https://doi.org/10.1515/9783110673241-002

Posted at the Zurich Open Repository and Archive, University of Zurich

ZORA URL: https://doi.org/10.5167/uzh-186693

Book Section

Published Version

Originally published at:

Dürscheid, Christa (2020). Zeichen setzen im digitalen Schreiben. In: Androutsopoulos, Jannis; Busch, Florian. Register des Graphischen : Variation, Interaktion und Reflexion in der digitalen Schriftlichkeit. Berlin, Boston: De Gruyter, 31-51.

DOI: https://doi.org/10.1515/9783110673241-002 


\section{Christa Dürscheid}

\section{Zeichen setzen im digitalen Schreiben}

\section{Vorbemerkungen}

Der vorliegende Beitrag befasst sich mit dem Schreiben an der Tastatur (digital) und nicht von Hand (analog). Hier aber stellt sich schon die erste Frage: Es ist ja auch möglich, mit einem Stift oder mit dem Finger auf einen Touchscreen zu schreiben. Handelt es sich dabei um ein analoges Schreiben (da von Hand) oder um ein digitales (da die derart eingegebenen Schriftzeichen vom Computer digital verarbeitet werden)? Weiter muss man bedenken, dass auch das Schreibmaschineschreiben ein Tastaturschreiben ist. Doch ist dies ein digitales Schreiben? Das ist sicher nicht der Fall. Denn auch wenn elektrische Schreibmaschinen weitaus mehr Möglichkeiten in der Textverarbeitung bieten als mechanische: Sie sind nicht computergesteuert und die Textdarstellung ist nicht digital. ${ }^{1}$ Die Fragen zeigen aber, dass es sich lohnt, einen Moment über den Terminus ,digitales Schreiben' nachzudenken, der heute überall wie selbstverständlich verwendet wird. Zwar steht in der Folge auch hier - wie in den meisten Beiträgen dieses Bandes nur ein Typus dieses digitalen Schreibens im Fokus, das interaktionsorientierte Schreiben (s.u.); in einem ersten Schritt aber soll der Terminus in seiner ganzen Breite betrachtet werden.

In Abschn. 2 wird zunächst erläutert, worin die Unterschiede zwischen dem digitalen und dem analogen Schreiben bestehen und welche Vorteile das jeweilige Schreiben bietet. Den Schwerpunkt lege ich dabei auf den Schreibprozess und nicht auf das Schreibprodukt. So kann es durchaus sein, dass ein Text zwar digital-schriftlich erscheint, dass er aber - wie z.B. beim Diktieren einer E-Mail über die Spracherkennung am Handy der Fall - nicht geschrieben wurde. Solche Beispiele klammere ich aus, da die Zeicheneingabe nicht schriftlich erfolgt. In Abschn. 3 wende ich mich der Verwendung von Bildzeichen (Emojis) zu, in Abschn. 4 der Verwendung von Interpunktionszeichen; hier werden einige grundsätzliche Überlegungen zu beiden Zeichentypen angestellt. Auf Emojis beziehe

1 Man mag sich fragen, ob Schreibmaschinen überhaupt noch im Einsatz sind, ob sie also eigens erwähnt werden müssen, wenn im Jahr 2018 vom Tastaturschreiben die Rede ist. Tatsächlich ist ihre Nutzung seit den 1980er Jahren stark zurückgegangen (vgl. Dingwerth 2008), es gibt aber immer noch einige wenige Bereiche, in denen sie zum Einsatz kommen (z.B. zum Ausfüllen von Formularen und Überweisungsträgern, zum Beschriften von Briefumschlägen). 
ich mich, weil ihr Auftreten als ein charakteristisches Merkmal der digitalen Schriftlichkeit angesehen wird (vgl. dazu aber weiter unten). Für die Interpunktionszeichen gilt das zwar nicht in gleicher Weise, diese kommen in allen Texten, ob analog oder digital, vor. Doch treten einige von ihnen (z.B. der Satzschlusspunkt) im digitalen Schreiben in spezifischer Weise auf. In Abschn. 5 zeige ich am Beispiel von WhatsApp-Nachrichten, wie Emojis und Punkte gesetzt werden und welche Regularitäten dahinterstehen. Illustriert wird dies am Beispiel von Daten aus dem Schweizer WhatsApp-Korpus, das ca. 750'000 Nachrichten umfasst (vgl. Ueberwasser/Stark 2017). ${ }^{2}$ Abschließend wird die Frage gestellt, wie die Entwicklung in der WhatsApp-Kommunikation weitergehen wird (Abschn. 6). Wie gestaltet sich die Zeichen-Setzung im digitalen Schreiben in einigen Jahren?

\section{Digital schreiben - analog schreiben}

Wenn hier vom digitalen Schreiben die Rede ist, dann ist damit die Eingabe von graphischen Zeichen (z.B. Buchstaben, Ziffern, Sonderzeichen) an der Computertastatur gemeint. Diese Eingabe vollzieht sich in der Regel über eine physische Tastatur (z.B. an einem Desktop) oder über ein virtuelles Tastaturfeld, das auf dem Bildschirm bedient wird (z.B. am Tablet). Möglich ist aber auch, dass der Text nicht Buchstabe für Buchstabe über die Tastatur eingegeben wird, sondern dass der Schreiber ${ }^{3}$ Wörter auswählt, die während des Tippens in einer schmalen Leiste eingeblendet werden. An dieser Stelle seines Textes schreibt er dann zwar nicht selbst, er entscheidet aber darüber, ob er den betreffenden Wortvorschlag annehmen oder ablehnen möchte. ${ }^{4}$ Da solche Wortvorschläge vom System nur

2 Das Projekt wird vom Schweizerischen Nationalfonds unterstützt (Fördernummer CRSII1_160714). Für weitere Informationen siehe <www.whatsup-switzerland.ch> (06.12.2019).

3 Ich verwende im vorliegenden Beitrag aus Gründen der besseren Lesbarkeit das generische Maskulinum. Diese Fußnote möchte ich auch dazu nutzen, der Person zu danken, die meine Arbeit seit Jahren in ein perfektes Layout bringt: Andi Gredig. Mein Dank gilt außerdem den Bandherausgebern für ihre wertvollen Hinweise zum Text und dem Alfried Krupp Kolleg in Greifswald, wo die Endversion des vorliegenden Beitrags entstanden ist.

4 Manche Schreiber verfassen den Text sogar so, dass der Computer möglichst viele Wortvorschläge anbieten kann (indem sie z.B. auf Dialektschreibung verzichten). Es stellt sich dazu die Frage, wer der eigentliche Akteur des Schreibens ist. Ist es der Computer, weil dieser im Kontext passende Wortvorschläge macht (vgl. dazu auch Abschn. 3)? Oder ist es der Mensch, da er es ist, der das Wort auswählt? Diese Frage kann hier nicht diskutiert werden; es sei aber darauf hingewiesen, dass Henning Lobin in seinem Buch „Engelbarts Traum“ auf anschauliche Weise darlegt, wie der Computer uns Lesen und Schreiben abnimmt (so der Untertitel). Dieses Buch 
dann generiert werden, wenn bereits Text über die Tastatur eingegeben wurde, zähle ich auch dies zum digitalen Schreiben. Das Schreiben mit einem Stift oder dem Finger auf dem Touchscreen gehört aber nicht dazu, der Schreibprozess vollzieht sich in diesem Fall handschriftlich, auch wenn das Schreibprodukt digital vorliegt. Wichtig ist weiter anzumerken, dass das digitale Schreiben nicht immer in Verbindung mit dem Internet steht. So kann ein Text offline eingegeben werden - und dies sowohl an einem stationären Gerät als auch von unterwegs (z.B. am Handy). Auch in diesen Fällen handelt es sich um ein digitales Schreiben.

Vergleichen wir damit nun das analoge Schreiben (siehe dazu auch Böhm/Gaetje 2014). Prototypisch steht dafür das Schreiben von Hand auf Papier - und damit ein Schreiben, das bereits als „old school“ bezeichnet wird. ${ }^{5}$ Gegenwärtig gibt es nur noch wenige Bereiche, in denen auf diese Weise geschrieben wird (vgl. Gredig 2019). Das Schreiben von Liebesbriefen und Kondolenzbriefen mag noch dazu gehören, oft sind es aber nur noch Einkaufslisten, Notizzettel oder Grußkarten aus den Ferien, die handschriftlich verfasst werden. Vor diesem Hintergrund mutet es geradezu anachronistisch an, wenn heute noch in der Schule und an der Universität Klausuren von Hand geschrieben werden (z.B. im Abitur, in Bachelor-Abschlussprüfungen). Doch vermutlich wird sich das durch den zunehmenden Einsatz digitaler Technologien bald ändern, so dass auch diese Bastion des analogen Schreibens in Schule und Hochschule bald fallen wird. ${ }^{6}$ Weiter ist zu vermuten, dass Multiple-Choice-Tests (wie z.B. im Medizinstudium) oder Befragungen im Kontext von Datenerhebungen (wie z.B. in der Psychologie), die noch im Paper-Pencil-Verfahren durchgeführt werden, künftig durch digitale Varianten ersetzt werden. Auf diese Weise lassen sich Testunterlagen resp. Fragebogen schneller auswerten, da die erhobenen Daten direkt in eine

erschien im Jahr 2014; heute ist die Entwicklung noch weiter fortgeschritten (vgl. dazu den Diskussionsbeitrag von Gerd Antos mit dem Titel Wenn Roboter „mitreden“, siehe aber auch die kritische Stellungnahme von Wolfgang Krischke in der Frankfurter Allgemeinen Zeitung vom 10.1.2018: „Mit Robotern reden“).

5 Vgl. dazu den folgenden Dialog auf der Website <http://www.gutefrage.net/frage/wie-ist-eingedicht-wenn-es-analog-geschrieben-ist> (08.12.2019): Die ,gute Frage` lautete (Wiedergabe im Original): „Hallo Leute, wir sollen für den Deutschunterricht ein analoges Gedicht schreiben. Leider bin ich in sowas sehr dumm und wüsste gerne wie ein Gedicht geschrieben ist, wenn es analog ist!!“ Als Antwort erhielt der Schüler u.a. den folgenden Kommentar: „Na eben nicht Digital auf dem Rechner sondern handgeschrieben auf einem Blatt Papier. Eben Oldschool.“

6 Vgl. dazu die Beiträge auf der Plattform <www.digitalisierung-bildung.de> (08.12.2019). Hier sind Blogbeiträge, Literaturhinweise und Hintergrundinformationen rund um das Thema Digitalisierung in Schule, Hochschule und beruflicher Weiterbildung zusammengestellt. 
Auswertungssoftware eingelesen werden. Beim analogen Schreiben sind dagegen zusätzliche Arbeitsschritte erforderlich.

Doch hat das analoge gegenüber dem digitalen Schreiben nicht nur Nachteile, sondern auch einige Vorteile - und dies nicht nur im Hinblick darauf, dass das Handschreiben unabhängig vom Einsatz eines Computers, unabhängig von funktionierender Software und unabhängig von der Stromversorgung ist. Ein Vorteil besteht darin, dass der Schreiber nicht auf das Zeicheninventar angewiesen ist, das ihm der Computer zur Verfügung stellt. Handschriftlich kann er beliebig variieren, den Text mit Smileys oder anderen Zeichen verzieren, die Buchstaben in verschiedenen Größen und Farben zu Papier bringen und verschiedene Handschriften ausprobieren. Grenzen sind ihm hier durch seine zeichnerischen Fertigkeiten und die zur Verfügung stehenden Schreibutensilien gesetzt, nicht aber durch die Technik. Oder anders gesagt: Zwar spielen auch für das Handschreiben die jeweiligen Rahmenbedingungen eine Rolle (z.B. die Farbe und Druckstärke der Stifte, die Qualität des Papiers), es sind dies aber andere Affordanzen als im digitalen Schreiben.

Auf der anderen Seite bietet die Tatsache, dass es am Computer eine Vielzahl von Optionen zur Textgestaltung gibt, gegenüber dem chirographischen Schreiben große Vorteile - und dies sowohl auf der Makro-, der Meso- als auch der Mikroebene des Textes. ${ }^{7}$ Was die Mikroebene betrifft, so zeigen sich diese Vorteile nicht nur in den verschiedenen Möglichkeiten der Schriftgestaltung, sondern auch in der Auswahl an Schriften (im Sinne von: Notationssystemen). Denn im Prinzip können alle alphabetischen und nicht-alphabetischen Zeichen, für die im internationalen Unicode-Standard ein digitaler Code (z.B. U+0566) festgelegt ist, eingegeben werden. Einschränkungen sind nur dadurch gegeben, dass die Software nicht vorhanden ist oder der Schriftfont fehlt, in dem das Zeichen dargestellt werden kann. ${ }^{8}$ Ist dies aber der Fall, dann ist es dem Schreiber umstandslos möglich, in seinen Text chinesische Schriftzeichen, ägyptische Hieroglyphen, koreanische Hangul-Zeichen oder kyrillische Buchstaben einzufügen, auch wenn

7 Zur Mikrotypographie zählen nach Stöckl (in Auswahl) die Schriftart und die Schriftgröße, zur Mesotypographie der Zeichenabstand, der Wortabstand, der Zeilenabstand und die Ausrichtung des Textes (z.B. Blocksatz), zur Makrotypographie die Gestaltung der Absätze, Einrückungen und typographische Hervorhebungen (vgl. Stöckl 2004: 22-23).

8 So kann es sein, dass ein Unicode-Schriftzeichen am Computer nicht angezeigt wird, weil in dem Font, in dem man schreibt (z.B. Times), dafür keine grafische Repräsentation vorgesehen ist. Dann kann es passieren, dass das Textverarbeitungsprogramm auf eine Schrift ausweicht, in der das Zeichen darstellbar ist (z.B. Tahoma) - allerdings ohne dies im Schriftmenü transparent zu machen (Andi Gredig, p.c.). 
er das jeweilige Schriftsystem nicht beherrscht und die Schriftzeichen ohne Übung nicht von Hand darstellen könnte.

Über den Unicode-Zeichensatz stehen am Computer aber nicht nur alle Schriftzeichen aus allen gängigen Schriftsystemen früherer und heutiger Zeit zur Verfügung, sondern auch eine andere Klasse von Zeichen: die Emojis (z.B. $P$, 8). Derzeit sind 3'187 Emojis im Unicode-Zeichensatz gelistet, ${ }^{9}$ nach jeweils sorgfältiger Prüfung durch das Unicode-Konsortium kommen jedes Jahr neue hinzu (vgl. Dürscheid 2018). Zwar mag man einwenden, dass auch der ,Handschreiber' solch kleine Bildchen von Verkehrsmitteln, Sportgeräten, Speisen oder Freizeitbeschäftigungen in seinen Text einfügen und farbig gestalten kann, sofern er über sehr gute Zeichenkenntnisse verfügt. De facto wird ihm dies aber kaum gelingen, zumal die Bilder, wenn sie emoji-ähnlich aussehen sollten, dieselben Proportionen wie Schriftzeichen haben müssten, was wiederum ein besonders großes zeichnerisches Geschick erfordert. Denn ein wesentliches Kennzeichen von Emojis ist, dass sie nicht separat als Bilder zum Text hinzugefügt werden (wie dies bei den sog. Stickern der Fall ist), sondern in einer Reihe mit Schriftzeichen stehen (also z.B. mit den Buchstaben des lateinischen Alphabets) und in etwa die Größe von Buchstaben haben - und das lässt sich chirographisch sehr schwer darstellen. Dies illustriert das folgende, konstruierte Beispiel: Ich

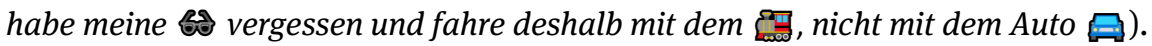
Will man die drei Bilder in diesem Satz in die Buchstabensequenz einbetten, dann wird das handschriftlich vermutlich einige Zeit in Anspruch nehmen. Beim Tastaturschreiben dagegen genügt es, die Zeichen über eine Tastenkombination (ALT-Taste + Zeichencode) oder auf der virtuellen Tastatur einzugeben oder das entsprechende Menu aufzurufen, in dem die Zeichen ausgewählt werden können.

\section{Bildzeichen}

In Anlehnung an die Übersetzung von $e$ (japanisch für ,Bild‘) und moji (japanisch für ,Buchstabe, Schriftzeichen') verwende ich als Synonym für das Wort Emoji hier die deutsche Bezeichnung Bildzeichen (wobei es streng genommen Bildschriftzeichen heißen müsste). Viele Emojis sind symbolische Zeichen (z.B. die Währungszeichen-Emojis), andere stehen in einer Ähnlichkeitsbeziehung zum Dargestellten (z.B. die Transportmittel-Emojis). Manche Emojis weisen eine

9 Vgl. unter <unicode.org/emoji/charts/emoji-counts.html> (06.12.2019). 
größere Ähnlichkeit zum Dargestellten auf (z.B. das Geschenk-Emoji oder das Kleeblatt-Emoji), andere sind stark schematisierte Darstellungen (z.B. die Gesichtszeichen-Emojis). Grundsätzlich lassen sich die Bildzeichen sowohl als ikonische Zeichen als auch als Symbole verwenden. So steht in dem Satz Ich habe gestern ein gefunden das Kleeblatt-Emoji ikonisch für das betreffende Objekt; es tritt in Referenzfunktion auf (vgl. Dürscheid/Frick 2016). Ergänzt der Schreiber dagegen den Satz Ich wünsche dir viel Glück um das Kleeblatt-Emoji oder setzt er nach einer Aussage wie Ich finde das toll das Daumenhochzeichen, um seine Aussage zu bekräftigen, dann haben diese Emojis symbolischen Charakter. Die Bedeutung der Bildzeichen kann in diesen Fällen nur über Regeln hergeleitet werden, sie lässt sich nicht über die bildhafte Darstellung erschließen.

Wie bereits erläutert, ist die Verwendung von Emojis ein Merkmal des digitalen, nicht-analogen Schreibens, im Prinzip können sie also in allen Texten und allen Textsorten auftreten, die am Computer geschrieben werden. In der (Schreib-)Praxis kommen sie aber nur in einer kleinen Klasse von Texten vor. Meist handelt es sich dabei um Texte, die in einem informellen Duktus stehen, die zum einen also konzeptionell mündlich sind und zum anderen, wie Angelika Storrer es in ihren Arbeiten nennt, für ein ,interaktionsorientiertes Schreiben' stehen. ${ }^{10}$ Diese beiden Aspekte sind unabhängig voneinander zu betrachten: Nicht alle konzeptionell mündlichen Texten sind interaktionsorientiert und nicht alle interaktionsorientierten Texte sind konzeptionell mündlich. Texte in Reiseblogs beispielsweise (vgl. dazu Klemm 2016) haben meist konzeptionell mündlichen Charakter, der Leser kann aber nicht unmittelbar darauf Bezug nehmen - und der Schreiber erwartet das auch nicht. ${ }^{11}$ Sie sind also nicht interaktionsorientiert. Doch kann dieses Schreiben interaktionsorientiert werden, wenn in dem Blog die Kommentarfunktion rege genutzt wird und der Blogger sich an der dialogischen

10 Vgl. dazu das folgende Zitat von Storrer (2014: 172): „Beim interaktionsorientierten Schreiben steht nicht das Schreibprodukt, sondern die laufende Interaktion im Mittelpunkt, deshalb kann die für das textorientierte Schreiben maßgebliche Tugend des Überarbeitens, des Feilens am Text, in den Hintergrund treten; die Schnelligkeit der Reaktion kann für den kommunikativen Erfolg wichtiger werden als die Prägnanz und Elaboriertheit des sprachlichen Ausdrucks.“ Dieser Art des digitalen Schreibens stellt Storrer das textorientierte Schreiben gegenüber (vgl. auch Storrer 2018). Dabei handelt es sich aber um zwei Pole eines Kontinuums; innerhalb eines längeren Nachrichtenaustauschs (z.B. per E-Mail) kann es zu Verschiebungen kommen.

11 Im Blog zu einer Marokko-Reise steht z.B. der folgende Eintrag: „Er ist Haschischbauer. Guck an. Er führt uns also hinauf in sein Dorf. Noch höher. Und dabei hatte ich mich gerade vom Aufstieg erholt“ (<https://www.reisedepeschen.de/bei-den-haschischbauern-im-rif/> (06.12.2019)). Das Beispiel weist Merkmale konzeptioneller Mündlichkeit auf (so z.B. die umgangssprachliche Ausdrucksweise „Guck an“). 
Kommunikation beteiligt. Ein Schreiben ist also nicht per se text- oder interaktionsorientiert, es kann sich im Laufe der Kommunikation verändern. Das trifft auch auf E-Mail-Korrespondenzen in offiziellen Kontexten zu. Stehen die Schreiber längere Zeit in einem schriftlichen Kontakt und gehen die Nachrichten in immer kürzer werdenden Abständen hin und her, dann kann das Schreiben interaktionsorientiert werden - und dann wird möglicherweise auch das eine oder andere Emoji gesetzt. Dies wiederum kann einen Akkommodationsprozess nach sich ziehen: Wenn der eine beginnt, Emojis zu verwenden, dann tut der andere das möglicherweise auch. Und das gilt natürlich auch umgekehrt. So ist in den „Ratschläge[n] aus der Geschäftswelt“, die auf der Internetsite Jobguide publiziert werden, Folgendes zu lesen:

Wer von seinem Gegenüber spezielle Vorlieben, den Geburtstag, den Urlaub etc. kennt, kann diese Tage mit den entsprechenden Emojis begleiten. Das zeigt, dass man aufmerksam ist und Anteil nimmt. Antwortet ein Gegenüber stets ohne Emojis und streng nach Vorschrift, sollte man in Zukunft auf jegliche Bildchen verzichten. Einen Versuch war es wenigstens wert.

<https://www.jobguide.de/news/nicht-ohne-meinen-emoji/> (06.12.2019)

Nach diesen allgemeinen Bemerkungen zum digitalen Schreiben lege ich nun den Schwerpunkt nur noch auf solche Texte, die in der privaten, informellen Alltagskommunikation ausgetauscht werden, die tendenziell also konzeptionell mündlich sind und in einem interaktionsorientierten Kontext stehen (z.B. die Textnachrichten in einem WhatsApp-Familienchat). Die für solche Texte charakteristischen Merkmale habe ich bereits an anderer Stelle ausführlich beschrieben (vgl. Dürscheid/Frick 2016: 73-106), deshalb seien hier nur einige dieser Merkmale genannt: Auf lexikalisch-grammatischer Ebene treten z.B. elliptische Strukturen auf, es kommen Gesprächspartikeln und umgangssprachliche Ausdrücke vor, durchgängige Dialektschreibung ist möglich (z.B. in der Schweiz). Auf graphischer Ebene kann es zu Flüchtigkeitsfehlern kommen, systematische Abweichungen von der Standardorthographie (z.B. Substantivkleinschreibungen, Rebusschreibungen wie gute N8 und nicht-usualisierte Akronymschreibungen wie z.B. WE für Wochenende) treten auf, und auch die Wiederholung von Frage- und Ausrufezeichen ist ein typisches Merkmal solcher Texte. Androutsopoulos (i.d.B.) spricht in diesem Zusammenhang denn auch treffend von einer inflationären Verwendung von Interpunktionszeichen, die dazu führt, dass die Iterationen (z.B. von Ausrufezeichen) „pragmatisch normalisiert werden“ (vgl. dazu auch Androutsopoulos 2018).

Alle diese Merkmale wurden aber auch schon in früheren Arbeiten zur computervermittelten Kommunikation (CMC) genannt (vgl. Bieswanger 2013); sie sind nicht spezifisch für neuere Entwicklungen der digitalen Kommunikation. 
Was aber neu ist und in der linguistischen Forschung inzwischen immer mehr Beachtung findet (vgl. z.B. Danesi 2017), ist die Verwendung von Emojis. ${ }^{12}$ Neu ist auch ein anderes Merkmal, auf das in der CMC-Forschung bisher noch nicht aufmerksam gemacht wurde: das Setzen von Leerzeichen an der Komposita-Fuge. In der früheren SMS-Kommunikation, in der die Zeichenzahl pro SMS begrenzt war, wurde häufig auf Leerzeichen verzichtet, um Zeichen einzusparen (z.B. Kommespäter), heute ist das Gegenteil zu beobachten: Leerzeichen werden gesetzt, damit das System Wortvorschläge machen kann und man sich dadurch Tippaufwand spart. Man orientiert sich also an den Möglichkeiten, die der Computer bereitstellt, um das Wort nicht in voller Länge tippen zu müssen - auch dies ein Beispiel dafür, wie der Computer uns das Schreiben abnimmt (vgl. den Untertitel des Buches von Lobin 2014, siehe auch Lobin 2018). Vergegenwärtigen wir uns dies kurz an einem Beispiel: Das Apple-Betriebssystem iOS 10.3.3 kennt als Wortvorschlag zwar das Kompositum Perlenkette, nicht aber das Wort Perlenschnur. Will man nun in einer Textnachricht schreiben, dass die Perlenschnur gerissen ist, hat man drei Möglichkeiten:

1. Man tippt das Wort Perlenschnur Buchstabe für Buchstabe ein.

2. Man akzeptiert den Wortvorschlag Perlenkette und damit ein Wort, das man eigentlich nicht verwenden wollte.

3. Man tippt $p$-e-r-l, akzeptiert den Wortvorschlag Perlen, setzt ein Leerzeichen, beginnt mit dem Tippen des Wortes Schnur und hofft auf den Wortvorschlag Schnur. Dieser erscheint dann auch (allerdings erst nach der Eingabe der Buchstaben $s-c-h-n-u)$. Im Resultat steht das Kompositum Perlenschnur, doch mit (falschem) Leerzeichen: Perlen Schnur. Ich vermute, dass viele Komposita-Getrenntschreibungen in der Smartphone-Kommunikation auf dieses Verfahren zurückgehen; eine empirische Untersuchung dazu steht aber noch aus.

Halten wir fest: Es gibt (alte und neue) Merkmale, die charakteristisch für das digital-informelle Schreiben sind, diese treten aber nur unter bestimmten Bedingungen und in bestimmten Textsorten auf. Denn natürlich lassen sich über alle Textsorten hinweg im digitalen (wie auch im analogen) Schreiben viele

12 Was in der Forschung bislang auch kaum beachtet wird, ist der Einsatz von Emojis in Rebusschreibungen. So weiß ich aus Gesprächen mit Studierenden, dass das Wort nice, das in der Wahl zum Anglizismus des Jahres 2017 den dritten Rang belegte (siehe unter $<$ http://www.anglizismusdesjahres.de/anglizismen-des-jahres/adj-2017/> (06.12.2019)), gelegentlich mit dem Eiscreme-Emoji dargestellt wird (vgl. $n \hat{\theta}$ ). Ein anderes Beispiel hierfür ist @steig (Julien Reimer, p.c.). Dabei handelt es sich vermutlich um graphostilistische Spielereien; auf jeden Fall sind solche Schreibweisen hochgradig markiert. 
verschiedene Schreibregister unterscheiden (vgl. Schubert/Sanchez-Stockhammer 2014), es muss deshalb immer präzisiert werden, welche Textsorten gemeint sind, wenn einschlägige Merkmale informellen Schreibens (wie z.B. die Verwendung von Emojis) thematisiert werden. Texte, die in einem offiziellen Kontext stehen (z.B. geschäftliche E-Mails, Kündigungsschreiben), weisen diese Merkmale in der Regel nicht auf, von dem digitalen Schreiben kann also nicht die Rede sein. Es ist deshalb auch irreführend, wenn ein Buch, das die Auswirkungen digitaler Technologien auf den Sprachgebrauch beschreibt, den Titel „Digilect“ trägt (vgl. Veszelszki 2016). Im Buch selbst wird diese Bezeichnung zwar problematisiert, und auch in den Verlagsinformationen wird der Eindruck relativiert, das digitale Schreiben lasse sich als homogene Einheit beschreiben. ${ }^{13}$ Durch den Titel drängt sich aber die Vermutung auf, es gebe einen Schreibstil, der sich über alle digitalen Textsorten hinweg erstrecken würde. Das ist falsch. So wenig, wie es eine Netzsprache gibt (vgl. Dürscheid 2004), so wenig gibt es einen Digilekt. Man muss denn auch mit Karina Frick, die das Buch rezensiert hat, feststellen, dass es ,beinahe etwas anachronistisch an[mutet], einem Band über die Merkmale und Auswirkungen des digitalen Schreibens (im Internet) heutzutage noch diesen Titel zu geben“ (Frick 2018: 1).

\section{Interpunktionszeichen}

Interpunktionszeichen gehören - anders als Bildzeichen - zum Standardrepertoire des deutschen Schriftsystems; sie kommen über alle Textsorten hinweg vor. Doch gibt es auch hier spezifische Verwendungsweisen, die charakteristisch für Texte aus der privaten Alltagskommunikation sind. Auf den inflationären Gebrauch von Frage- und Ausrufezeichen wurde weiter oben schon verwiesen; ein anderes Merkmal ist, wie Androutsopoulos (i.d.B.) es nennt, die „Pragmatikalisierung“ syntaktischer Zeichen (Punkt, Auslassungspunkte). Ob es berechtigt ist, von einer solchen Pragmatikalisierung zu sprechen, wird weiter unten diskutiert (vgl. Abschn. 5); zunächst sei hier kurz erläutert, welche Typen von Zeichen zu den Interpunktionszeichen zählen und welchen Status die Interpunktion im deutschen Schriftsystem hat. Im Metzler Lexikon Sprache steht unter dem Eintrag ,Interpunktion“ die folgende Definition (in Auszügen):

13 Vgl. dazu das folgende Zitat auf der De-Gruyter-Website: „Naturally, the book presents the characteristics of the digital language variety (and coins the term digilect) but only to give a framework to the impact analysis“ <https://www.degruyter.com/view/product/476889> (31.01.2020). 
Interpunktion (lat. interpunctum >durch Punkte abgetrennt $\triangleleft$ Auch: Zeichensetzung. [...] I.zeichen bilden ein Inventar graph. Mittel, dessen Elemente in Satzzeichen und Wortzeichen eingeteilt werden können. Satzzeichen sind I.mittel, die über der Ebene von Sätzen und Satzteilen operieren. Wortzeichen (nicht mit Begriffszeichen und Ideogramm zu verwechseln) korrespondieren mit der Kategorie der freien Morpheme.

Glück/Rödel (2016: 306)

Interpunktion wird hier - anders als dies z.B. bei Nerius et al. (2007: 236-239) der Fall ist - als Oberbegriff angesehen; er steht nicht nur für das Setzen von Satzzeichen, sondern auch von sog. Wortzeichen. Ein solches Wortzeichen ist z.B. der Ergänzungsstrich. Er substituiert i.d.R. frei vorkommende Morpheme (vgl. aber fehler- und mangelhaft), wobei sich das Wortmaterial, das durch den Strich ersetzt wird, in unmittelbar vorangehender oder nachfolgender Umgebung befindet (vgl. be- und entladen; bergauf und -ab). ${ }^{14}$ Wie der Ergänzungsstrich zählen z.B. auch die Auslassungspunkte zu den Interpunktionszeichen; diese werden, so liest man im Zweifelsfälle-Duden, dann gesetzt, „wenn eine Rede abgebrochen oder ein Gedankenabschluss verschwiegen wird“ (Duden 2016: 118). Doch können Auslassungspunkte, wie andere Zeichen auch, im informell-digitalen Schreiben noch andere Funktionen aufweisen (vgl. dazu Androutsopoulos i.d.B.), die nicht diesen Vorgaben entsprechen. Dann stehen sie in Opposition zur Standardorthographie und entfalten möglicherweise gerade dadurch ihr kommunikatives Potential.

Für alle Interpunktionszeichen gilt, dass ihre Verwendung in der Orthographie des Deutschen verbindlich geregelt ist. Mehr noch: Im Deutschen ist sie sogar kodifiziert, d.h. in einem Amtlichen Regelwerk (im Folgenden: AR) niedergeschrieben. Dieses Regelwerk besteht aus einem Regelteil und einem Wörterverzeichnis und basiert auf den Empfehlungen des Rats für deutsche Rechtschreibung (siehe unter <http://www.rechtschreibrat.com/>). Die Setzung von Interpunktionszeichen wird hier im Kapitel zur „Zeichensetzung“ behandelt, doch ohne dass der Terminus Interpunktion überhaupt erwähnt würde. In den „Vorbemerkungen“ zu diesem Regelbereich wird zunächst auf die Satzzeichen Bezug genommen. Hier ist zu lesen, dass diese Grenz- und Gliederungssignale seien (vgl. AR 2018: 74), dann werden die Satzzeichen aufgelistet (Punkt, Ausrufezeichen, Fragezeichen, Komma, Semikolon, Doppelpunkt, Gedankenstrich, Klammern, Anführungszeichen) und von anderen Zeichen unterschieden, die zur „Markierung von Auslassungen“ (Apostroph, Ergänzungsstrich, Auslassungspunkte) oder „zur Kennzeichnung der Wörter bestimmter Gruppen“ (Punkt nach

14 Gelegentlich kommt es allerdings vor, dass der Ergänzungsstrich vergessen wird und das zu einer nicht-intendierten Lesart führt (vgl. Fenster und Gebäudereinigung). 
Abkürzungen bzw. Ordinalzahlen, Schrägstrich) dienen (AR 2018: 74). Der Bindestrich wird in dem Kapitel nicht erwähnt, ihm ist ein eigener Regelbereich gewidmet (Kap. C: Schreibung mit Bindestrich). Das gilt auch für den Trennstrich (Kap. F: Worttrennung am Zeilenende). Wie sinnvoll die Auslagerung dieser beiden Interpunktionszeichen aus dem Kapitel zur Zeichensetzung ist, soll hier nicht diskutiert werden; es sei dazu aber auf die Arbeit von Bredel hingewiesen, die dies kritisch kommentiert (vgl. Bredel 2008: 72-73).

Ursula Bredel listet in ihren Arbeiten zur Interpunktionstheorie die folgenden Interpunktionszeichen auf: Punkt, Doppelpunkt, Fragezeichen, Ausrufezeichen, Komma, Semikolon, Klammern, Anführungszeichen, Divis, Apostroph, Auslassungspunkte und Gedankenstrich. ${ }^{15}$ Von diesen zwölf Zeichen unterscheidet sie weitere Zeichentypen (z.B. die Sonderzeichen), die sie auf der Basis binärer Merkmale klassifiziert. Das zeigt die folgende Tabelle, die von Bredel (2008) übernommen wurde. ${ }^{16}$

Tab. 1: Binäre Merkmalsklassifikation nach Bredel (2008: 23)

\begin{tabular}{lcccccc} 
& Diakritika & Buchstaben & Ziffern & Sonderz. & IP-Zeichen & Leerzeichen \\
\hline darstellbar & + & + & + & + & + & - \\
\hline verbalisierbar & - & + & + & + & - & - \\
\hline kombinierbar & - & + & + & - & - & - \\
\hline zweielementig & - & + & - & - & - & - \\
\hline additiv & + & - & - & - & - & - \\
\hline
\end{tabular}

Diese Klassifikation sei hier kurz erläutert, da sie sich auch auf Emojis beziehen lässt: Leerzeichen beispielsweise tragen das Merkmal [-darstellbar], d.h. man erkennt sie nur dann, wenn sie in einer graphischen Umgebung auftreten. Das Merkmal [+additiv] unterscheidet die Diakritika von allen anderen Zeichen. Diakritika kommen als segmentale Mittel additiv zu einem Zeichen hinzu, sie treten aber nie selbständig auf. ${ }^{17}$ Weiter sind Diakritika nicht verbalisierbar, was bei

15 Divis ist der typographische Terminus für den Viertelgeviertstrich. Er steht für den Ergänzungs-, den Binde- und den Trennstrich.

16 Bredel bezieht sich hier nur auf den alphabetischen Schrifttyp. Für syllabische und logographische Schriften gilt diese Klassifikation nicht.

17 Im Deutschen gehört dazu das Trema, das den Umlaut anzeigt. Zu den Umlautzeichen sei hier noch ein kleiner Exkurs gestattet: Auf einer deutschen Tastatur kann man diese über einen einzigen Tastendruck darstellen (bei Großbuchstaben in Kombination mit der Shifttaste). Für Schweizer Tastaturen gilt das nicht, nur die Kleinbuchstaben $\langle\ddot{a}\rangle,<\ddot{0}>$ und $<\ddot{u}>$ haben hier eine eigene Tastaturbelegung. Die Großbuchstaben $\langle\ddot{\mathrm{A}}\rangle,\langle\ddot{\mathrm{O}}\rangle$ und $\langle\ddot{\mathrm{U}}\rangle$ lassen sich aber über die 
Buchstaben, Ziffern und Sonderzeichen (z.B. $\langle \%\rangle,\langle \&\rangle$ ) der Fall ist. Buchstaben und Ziffern wiederum sind zu Einheiten größeren Typs kombinierbar (zu Wörtern und Zahlen), Sonderzeichen dagegen nicht. Alle Buchstaben treten im deutschen Schriftsystem zweielementig auf (als Majuskeln und Minuskeln), alle anderen graphischen Zeichen nicht. ${ }^{18}$

Was nun die Emojis betrifft, so gehören diese zwar nicht zum Schriftsystem des Deutschen, sie teilen mit den Zeichen, die Bredel (2008) in ihrer Tabelle auflistet, aber einige Merkmale. Das zeigt sich z.B. daran, dass sie als Ideogramme fungieren können (vgl. zu diesem Terminus Gallmann 1985). In dieser Verwendungsweise (vgl. Ich $\bigcirc$ dich) gehören sie zu den Sonderzeichen (z.B. $<\&>,<\%>$ ), die Gallmann (1985: 13) als eine formal definierte Klasse beschreibt, die prinzipiell erweitert werden kann. An anderer Stelle habe ich diese Überlegungen bereits dargelegt (vgl. Dürscheid/Siever 2017), deshalb sei hier nur so viel gesagt: Emojis können wie Sonderzeichen verwendet werden. Das umfasst aber nur einen kleinen Ausschnitt ihres Anwendungsspektrums, daneben weisen sie viele weitere Funktionen auf, auf die in der - inzwischen schon recht umfangreichen Literatur zu diesem Thema - Bezug genommen wird (z.B. Pappert 2017).

\section{Zeichen setzen in der WhatsApp- Kommunikation}

In diesem Abschnitt beziehe mich auf die Bachelor-Arbeit von Yvonne Zgraggen, die als temporäre Mitarbeiterin im Schweizer WhatsApp-Projekt Daten zur Interpunktion und zur Verwendung von Emojis ausgewertet hat. Sie legte ihrer Untersuchung das schweizerdeutsche Subkorpus zugrunde, das den größten Anteil im Gesamtkorpus ausmacht: Es beinhaltet 125 Chats, 479'031 Nachrichten und 1'820'316 Tokens (Angaben auf der Basis der Korpusversion 5.0, Release von Juli 2017). Zgraggen geht der Frage nach, wie häufig in diesem Korpus Emojis, Punkte, Fragezeichen und Ausrufezeichen vorkommen und wie diese distribuiert sind (z.B. beitragsfinal oder beitragsintern). Über die Funktionen, die diese

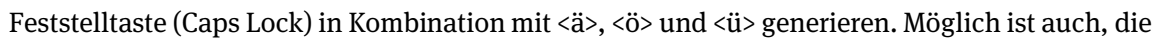
sog. Trema-Taste zu verwenden, die es wiederum in der deutschen Standard-Tastaturbelegung nicht gibt. Drückt man diese ,Zwei-Pünktchen-Taste“ und dann die Buchstaben $\langle\mathrm{A}\rangle,\langle\mathrm{O}\rangle$ oder $<\mathrm{U}\rangle$, dann wird der entsprechende Buchstabe erzeugt.

18 Bis Dezember 2017 galt diese Aussage nicht für das $\langle\beta\rangle$. Dieses Schriftzeichen war das einzige, das im deutschen Alphabet nur als Kleinbuchstabe geführt wurde. Mittlerweile gibt es aber auch das Eszett als Klein- und als Großbuchstabe (vgl. AR 2018). 
Zeichen-Setzungen haben, kann sie auf der Basis von solch quantitativen Auswertungen nichts aussagen, die Zahlen geben aber interessante Aufschlüsse zu der Frage, wie frequent bestimmte Zeichen (z.B. Fragezeichen) sind und an welchen Positionen sie stehen. Was den Satzschlusspunkt betrifft, so wird in der Literatur und auch in einschlägigen Medienberichten z.B. immer wieder darauf verwiesen, dass er am Ende einer Nachricht häufig fehle (vgl. Busch 2017). Kann das durch die Daten aus dem WhatsApp-Korpus bestätigt werden?

Dies ist eine der Fragen, die Zgraggen in ihrer BA-Arbeit untersuchte. Bevor ich ihre empirische Studie aber darstelle, sei zunächst etwas zur Punktsetzung im Allgemeinen gesagt: Der Punkt hat eine syntaktische Funktion, er zeigt an, dass die vorangehende Einheit abgeschlossen ist. Darin unterscheidet er sich vom Frage- und Ausrufezeichen, die „,neben ihrer Grenz- und Gliederungsfunktion noch eine oder mehrere kommunikativ-pragmatische Funktionen zu erfüllen haben“ (Nerius 2007: 242). Bredel (2008) beschreibt die syntaktische Funktion des Punkts mit den folgenden Worten:

Der Punkt [...] instruiert den Leser, weder phrasale noch lexikalische Informationen zu einer Auswertung verfügbar zu halten. Der Leser muss die syntaktische Verknüpfung abschließen und den syntaktischen Arbeitsspeicher leeren.

Bredel (2008: 191)

Nun erkennt man am Ende einer WhatsApp-Nachricht aber auch ohne einen Punkt, dass keine weiteren Informationen folgen. Ein Grenzsignal ist hier im Grunde nicht nötig, der „syntaktische Arbeitsspeicher“ wird ohnehin geleert, da der Text an dieser Stelle endet. Allein aus diesem Grund lässt sich vermuten, dass in der WhatsApp-Kommunikation der Punkt am Ende einer Nachricht oft fehlt. ${ }^{19}$ Das Nicht-Setzen eines Satzschlusspunktes ist im Übrigen auch in der Amtlichen Regelung vorgesehen. Hier heißt es auf S. 75: „Nach freistehenden Zeilen setzt man keinen Punkt“. Als Beispiel werden u.a. Überschriften genannt (vgl. „Schneeverwehungen behindern Autoverkehr“). Für diese gilt, dass sie kleine selbständige Einheiten darstellen und typographisch vom Rest des Textes abgesetzt sind - ein Punkt ist hier nicht nötig (und darf auch gar nicht gesetzt werden). Beides gilt strenggenommen auch für die einzelnen Beiträge in WhatsAppChats: Jeder Beitrag steht in einer separaten Sprechblase, es sind selbständige kleine Einheiten, die typographisch von den (möglicherweise) folgenden

19 Das freilich könnte auch für das Ende eines Buches gelten; warum also tritt dieses Phänomen im interaktionsorientierten Schreiben so häufig auf? An dieser Frage setzen soziolinguistisch ausgerichtete Arbeiten zur Interpunktion an, auf die ich weiter unten kurz eingehen werde. 
Sprechblasen abgesetzt sind. Ein Punkt ist also nicht nötig, um das Ende des Beitrags anzuzeigen.

Damit komme ich zu den oben bereits erwähnten Arbeiten zur Nicht-PunktSetzung, in denen vermutet wird, dass dem Punkt in der informellen Schriftlichkeit eine neue pragmatische Funktion zukommt. Diese Auffassung vertritt z.B. Busch (2017) in seinem Beitrag mit dem Titel „Zeichensetzung im digitalen Schreiben von Jugendlichen“, dem ein Korpus von 61 WhatsApp-Chats von norddeutschen Jugendlichen zwischen 12 und 19 Jahren zugrunde liegt. Busch stellt fest, dass der Punkt „,als syntaktisches Grenzsignal im informellen Schreiben eine Aufladung als sozialer Kontextualisierungshinweis erfährt, er gewinnt pragmatische Funktion“ (Busch 2017: 87). Bestätigt sieht er diese Annahme durch ein Experiment an der Universität Binghamton (USA), das auch in den Medien viel Aufmerksamkeit erregte. In diesem Experiment wurden 126 Teilnehmern handschriftliche und digitale Kurztexte mit und ohne Schlusspunkt am Ende des Textes vorgelegt (vgl. Gunraj et al. 2016); dann wurden die Probanden dazu befragt, wie die Nachricht auf sie wirken würde. Das Abstract zu dem Artikel, in dem die Ergebnisse dieses Experiments vorgestellt werden, fasst den Befund wie folgt zusammen. „When the exchanges appeared as text messages, the responses that ended with a period were rated as less sincere than those that did not end with a period“ (Gunraj et al. 2016: 1068).

Ob diese Interpretation zutrifft, soll hier nicht diskutiert werden; es kann in Bezug auf die Daten aus dem WhatsApp-Projekt aber eine Antwort darauf gegeben, ob am Ende der Nachrichten tatsächlich oft kein Punkt gesetzt wird. Dabei ist allerdings zu bedenken, dass der Punkt zwar fehlen mag, dass aber möglicherweise ein anderes Zeichen die beitragsfinale Position einnimmt: das Emoji (vgl. dazu auch Androutsopoulos 2018: 736-741). Die von Yvonne Zgraggen in ihrer Bachelorarbeit hierzu durchgeführten Korpusauswertungen ergaben, dass am Ende der WhatsApp-Nachrichten sehr häufig Emojis stehen; hier gibt es fast 70’000 Treffer (vgl. Zgraggen 2017: 29). Frage- und Ausrufezeichen rangieren weit dahinter, kommen beide aber häufiger vor als der Punkt. Sein Vorkommen in beitragsfinaler Position ist im Schweizerdeutsch-Subkorpus nur mit 4'106 Treffern belegt.

Die Zahlen bestätigen somit die Aussage von Busch (2017): Beitragsfinale Sätze stehen oft ohne Punkt. Damit wird aber noch nichts darüber ausgesagt, ob es zutrifft, dass ,jeder Punkt, der dennoch gesetzt wird, im Kontrast zum Normalfall als markiert gelesen werden kann“ (Busch 2017: 89). Dies führt uns zu einer grundsätzlichen Frage: Ist es, wie in der Literatur oft zu lesen, zutreffend, dass die Schreiber mit der Zeichen-Setzung Kontextualisierungshinweise geben (vgl. Auer 1986), dass sie also ,Zeichen setzen“ wollen, indem sie bestimmte Zeichen 
setzen (Emojis) und andere weglassen (Punkte) ${ }^{20}$ Und wie sollen diese Hinweise interpretiert werden? Um hierzu verlässliche Aussage machen zu können, muss man Informantenbefragungen durchführen, aus den Daten selbst kann man dies nicht herauslesen. Eine solche Befragung (aus Leserperspektive) wurde in dem oben bereits erwähnten Experiment von Gunraj et al. (2016) durchgeführt, doch lassen sich diese Ergebnisse nicht generalisieren. Die Probleme, die bei einer solchen Befragung auftreten können, möchte ich kurz an einem Beispiel erläutern. Ich wähle dazu eine Nachricht aus dem Schweizerdeutsch-Subkorpus:

\section{Zerst mal: HAPPY BIRTHDAY MINI SCHWESTER!!

Abb. 1: Emojis in beitragsinterner und beitragsfinaler Position

Am Ende dieser Nachricht steht ein Herz-Emoji und auch in der Nachricht selbst wird ein Herz-Emoji verwendet (hier in Kombination mit weiteren Emojis). Beide Male hat das Emoji die Funktion, die Aussage zu kommentieren. Als Grenzsignal fungiert es (wenn überhaupt) nur am Ende der Nachricht; beitragsintern kommt ihm diese Funktion nicht zu. Würde diese Chat-Nachricht auf den Leser anders wirken, wenn der Schreiber sie mit einem Punkt beendet hätte (Ich liebe und vermisse dich.)? Würde sie z.B. als „less sincere“ wahrgenommen werden, wie Gunraj et al. (2016: 1069) dies nennen? Welchen Effekt hätte die Aussage auf den

20 Vgl. dazu Busch (2017: 89): „Nachdem seine syntaktische Funktion als Grenzsignal am Beitragsende nicht mehr von Belang ist, wird der Punkt dafür frei, pragmatische Information zu tragen. Es kommt zu der eingangs vorgestellten Lesart des Punkts als Kontextualisierungshinweis (Hervorhebung C.D.) für emotionale Distanz [...].“ 
Leser, wenn ein Ausrufezeichen gesetzt worden wäre (Ich liebe und vermisse dich!). Und welche Assoziationen würden sich bei ihm einstellen, wenn weder ein Satzzeichen noch ein Emoji stünde (Ich liebe und vermisse dich)? Die Antworten auf diese Fragen müssen offenbleiben, auf jeden Fall aber gilt: Die Punktsetzung kann in einer schriftlichen Interaktion einen zusätzlichen Interpretationsrahmen eröffnen (vgl. Androutsopoulos 2018: 736) - und dies v.a. dann, wenn sie im Kontrast zur Standardorthographie oder zu anderen in dem jeweiligen Kontext üblichen Schreibweisen steht (was nicht dasselbe ist).

Wie Abb. 1 weiter zeigt, stehen die sieben Emojis, die hier beitragsintern als eine Sequenz auftreten, in Kombination mit zwei Ausrufezeichen. Wie häufig kommt es vor, dass Emojis in einer solchen Kombination auftreten, und wie häufig ist es der Fall, dass sie dem bzw. den Interpunktionszeichen folgen? Auch dazu hat Yvonne Zgraggen im Schweizerdeutsch-Subkorpus Suchabfragen durchgeführt. Sie stellt fest, dass im Schnitt jede dritte Nachricht in diesem Subkorpus ein Emoji enthält, dass aber nur rund 5\% der Emojis in Kombination mit Interpunktionszeichen auftreten. Was die Frage betrifft, ob diese Emojis vor oder nach den Interpunktionszeichen stehen, so „liefern die Daten auch eine relativ eindeutige Antwort: 87\% der Emojis stehen nach dem Interpunktionszeichen“ (Zgraggen 2017: 33). In welcher Funktion das Emoji im jeweiligen Kontext auftritt, kann durch solche Auszählungen aber nicht ermittelt werden. So ist es möglich, dass der Schreiber mit dem Emoji, das er nach dem Punkt setzt, die vorangehende Aussage kommentieren möchte (z.B. Ich freue mich. (-)) oder damit einen neuen Satz beginnen möchte (z.B. $\hat{\ddot{w}}^{3}$ scheint). In diesem konstruierten Beispiel erscheint das Emoji nicht in Kommentarfunktion, sondern in Darstellungsfunktion (vgl. zu diesem Terminus Dürscheid/Frick 2016: 105), es steht für die Nominalphrase die Sonne.

Abschließend sei noch ein weiteres Beispiel aus dem WhatsApp-Korpus angeführt, das in diesem Fall aus dem nicht-dialektalen Subkorpus stammt. Das Beispiel ist insofern interessant, als hier zwei Emojis auftreten, von denen das eine als Erstglied eines Kompositums verwendet wird, das andere zur Kommentierung am Ende des Beitrags steht. Die für Emoji-Verwendungen charakteristischen Funktionen, Kommentar- und Darstellungsfunktion, sind also kombiniert. Der beitragsfinale Punkt fehlt in dieser Nachricht. Das überrascht nicht; er ist folgt man der oben vorgetragenen Argumentation - nicht nötig: 


\section{Ich geh mal $x^{*}$ modus einschalten

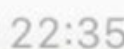

Abb. 2: Emojis in Darstellungs- und in Kommentarfunktion

Das Flugzeug-Emoji kann hier nicht als Flugzeug verbalisiert werden, obwohl man dies aufgrund der bildhaften Darstellung vielleicht meinen könnte. Denn wie der Kontext zeigt, entspricht dem Emoji ausdrucksseitig das Morphem Flug, das Kompositum steht also für Flugmodus. ${ }^{21}$ In anderen Fällen mag das Emoji die Wörter Flugzeug oder fliegen substituieren (wenn es z.B. in der Sequenz Ich fliege nach Rio anstelle des Verbs gesetzt würde). Daran sehen wir: Emojis können im Sinne Gallmanns (s.o.) zwar als Begriffszeichen (Ideogramme) verwendet werden, eine feste Ausdrucksseite haben sie aber nicht. Interessant wäre nun zu wissen, wie häufig eine solche Verwendungsweise (d.h. in Darstellungsfunktion) in den WhatsApp-Daten überhaupt auftritt. Da die WhatsApp-Nachrichten zu einer Zeit gesammelt wurden, als das System nur Wortvorschläge und noch keine Emoji-Vorschläge machte (im Jahr 2014), ist zu vermuten, dass diese Zahl gering ist. Eine genaue Angabe liegt im WhatsApp-Projekt aber nicht vor, die Abfrage gestaltet sich als sehr komplex. Hier zeigt sich ein grundsätzliches Problem: Quantitative Auswertungen sind in der empirischen Forschung zwar eine große Hilfe, oft aber ist zusätzlich eine manuelle Sichtung der Treffer erforderlich - und das ist je nach Datenmenge von Einzelpersonen kaum mehr zu bewältigen.

Abschließend sei dieses Problem noch an zwei weiteren Beispielen illustriert: Insgesamt finden sich im Schweizerdeutsch-Subkorpus 363 Treffer für das Semikolon und 3'384 Treffer für den Doppelpunkt. Die Zahlen sagen aber nichts darüber aus, wie oft diese Zeichen als Satzzeichen fungieren. Möglich ist ja auch, dass sie als Teile von Smileys vorkommen (z.B. ;-) oder :-)), sofern die Smileys auf der Basis von ASCII-Zeichen dargestellt werden - und das ist im Schweizer WhatsApp-Korpus noch häufig der Fall. Dasselbe Problem stellt sich bei den Auszählungen zum Divis. In welcher graphischen Funktion der Divis auftritt (ob z.B. als Ergänzungs- oder als Bindestrich), lässt sich nur feststellen, wenn die Belege nochmals systematisch überprüft werden. So führt die automatische Suche auch

21 Interessant ist das Beispiel auch deshalb, weil die meisten Smartphones den Flugmodus mit einem Icon anzeigen, das dem in Abb. 2 verwendeten Emoji sehr ähnlich ist (Florian Busch, p.c.). Streng genommen könnte das Kompositum also für das Wort Flugmodusmodus stehen. 
zu Nachrichten, in denen mathematische Gleichungen mit Divis stehen (vgl. den folgenden Treffer: Und isch 6yp+3y-12p-6 ned 3y(2p+1)-6(2p-1)). Darin zeigen sich die Herausforderungen in Zeiten von Big Data: Zwar ist es ist es möglich, große Textkorpora zu erstellen, doch in der Auswertung stößt man oft an die Grenzen der Machbarkeit. Hier sind wir in der Linguistik auf gute Parser angewiesen, die die systematische Abfrage nach bestimmten Zeichentypen bzw. Zeichenkombinationen in großen Datenmengen ermöglichen. Abgesehen davon kann aber auch eine punktuelle, qualitative Sichtung der Daten sehr aufschlussreich sein, und dies insbesondere dann, wenn man in einer Untersuchung zum interaktionsorientierten Schreiben nicht nur einzelne Nachrichten, sondern längere Chatsequenzen betrachten möchte.

\section{Ausblick und Zusammenfassung}

Der letztgenannte Punkt (ver-)führt mich dazu, einen Blick in die Zukunft zu werfen: Möglicherweise gestaltet sich die Datenauswertung in Zukunft ja einfacher, weil die Suchabfragen verfeinert und neue Abfrage-Tools entwickelt werden. In einigen Jahren dürfte es dann z.B. interessant sein, eine weitere Datensammlung zu WhatsApp (falls es diese Applikation noch gibt) durchzuführen, die Daten auf der Basis neuer Tools auszuwerten und die Ergebnisse mit den Daten aus dem Korpus von $2014 \mathrm{zu}$ vergleichen. Vielleicht wird sich in einer solch diachronen Studie zeigen, dass im Jahr 2024 mehr Emojis als im Jahr 2014 verwendet werden, und vielleicht ist es dann auch häufiger der Fall, dass Emojis in Darstellungsfunktion (also als Morphemersatz) auftreten. Denn es ist anzunehmen, dass die Emoji-Vorschläge, die das System heute anbietet, noch optimiert werden und dass es im Unicode-Zeichensatz in Zukunft noch weitaus mehr Emojis geben wird. Interessant wird es auch sein, auf der Basis großer Datenmengen zu überprüfen, ob Emojis in solchen Textsorten vorkommen, die weder interaktionsorientiert sind noch in einem informellen Kontext stehen (z.B. Werbeflyer, FirmenNewsletter). Diese Textkorpora lassen sich dann mit früheren Datensammlungen aus dem geschäftlichen Umfeld vergleichen.

Damit komme ich zum Schluss: Im Beitrag wurde gezeigt, welche Kennzeichen das digitale Schreiben (als Tätigkeit) aufweist und wo die Unterschiede zum analogen Schreiben liegen. Dann wurde das Augenmerk auf den Sprachgebrauch in digitalen Texten gerichtet und es wurde betont, dass es eine Vielzahl von Schreibregistern gibt, so dass die Rede von einem „Digilekt“ zu kurz greift. Im Anschluss daran wurde der Schwerpunkt auf Texte gelegt, die in einem interaktionsorientieren Kontext stehen und Merkmale konzeptioneller Mündlichkeit 
aufweisen. Ein Kennzeichen solcher Texte ist, dass die Schreiber - pauschal gesagt - bestimmte Zeichen (z.B. Emojis) setzen und andere nicht (z.B. Satzschlusspunkte). Um die beiden Zeichentypen im Schriftsystem des Deutschen einordnen zu können, folgten zunächst einige allgemeine Bemerkungen zur Verwendung von Bildzeichen und Interpunktionszeichen, dann wurden Daten aus dem Schweizer WhatsApp-Korpus herangezogen. Diese sollten Aufschluss über die Distribution von Bild- und Satzzeichen geben und die Aussagen mit quantitativen Korpusauswertungen abstützen. Abschließend wurde auf die Problematik solcher Auswertungen hingewiesen, die letztlich erst dann aussagekräftig sind, wenn sie nachbearbeitet werden. Dies führte zu einem Blick in die Zukunft und es wurde die Hoffnung zum Ausdruck gebracht, dass sich die Auswertung großer Textkorpora zur digitalen Alltagskommunikation künftig einfacher gestalten wird. Doch vielleicht wird es solche Textkorpora künftig auch nicht mehr geben, weil in der digitalen Alltagskommunikation das gesprochene Wort zu neuen Ehren kommt - man denke nur an die zunehmende Popularität von Sprachnachrichten oder an das Sprechen mit Alexa oder Siri.

\section{Literatur}

Amtliche Regelung der deutschen Rechtschreibung. 2018. Regeln und Wörterverzeichnis. Aktualisierte Fassung des Regelwerks entsprechend den Empfehlungen des Rats für deutsche Rechtschreibung 2016. <http://www.rechtschreibrat.com/DOX/rfdr_Regeln_2016_redi giert_2018.pdf> (06.12.2019).

Androutsopoulos, Jannis. 2018. Digitale Interpunktion: Stilistische Ressourcen und soziolinguistischer Wandel in der informellen digitalen Schriftlichkeit von Jugendlichen. In Arne Ziegler (Hg.), Jugendsprachen: aktuelle Perspektiven internationaler Forschung. Berlin \& Boston: de Gruyter, 721-748.

Androutsopoulos, Jannis. i.d.B. Auslassungspunkte in der schriftbasierten Interaktion.

Antos, Gerd. 2017. Wenn Roboter „mitreden“. Brauchen wir eine Disruptions-Forschung in der Linguistik? Zeitschrift für Germanistische Linguistik 45(3), 392-419.

Auer, Peter. 1986. Kontextualisierung. Studium Linguistik 19, 22-47.

Böhm, Manuela; Gätje, Olaf. 2014. Handschreiben - Handschriften - Handschriftlichkeit: Zu Praktik, Materialität und Theorie des Schreibens mit der Hand. Osnabrücker Beiträge zur Sprachtheorie (OBST) 85, 7-21.

Bieswanger, Markus. 2013. Micro-linguistic structural features of computer-mediated communication. In Susan C. Herring; Dieter Stein; Tuija Virtanen (Hgg.), Pragmatics of ComputerMediated Communication (Handbooks of Pragmatics 9). Berlin \& Boston: de Gruyter, 463485.

Bredel, Ursula. 2008. Die Interpunktion des Deutschen: Ein kompositionelles System zur Online-Steuerung des Lesens (Linguistische Arbeiten 522). Tübingen: Niemeyer. 
Busch, Florian. 2017. Informelle Interpunktion? Zeichensetzung im digitalen Schreiben von Jugendlichen. Der Deutschunterricht, Themenheft Soziolinguistik 4, 87-91.

Danesi, Marcel. 2017. The Semiotics of Emoji. The Rise of Visual Language in the Age of the Internet. London u.a.: Bloomsbury Publishing.

Dingwerth, Leonhard. 2008. Historische Schreibmaschinen - Geschichte, Technik und Faszination. Regenstauf: Battenberg-Verlag.

Duden - Das Wörterbuch der sprachlichen Zweifelsfälle. 2016. 8. Aufl. Berlin: Bibliographisches Institut.

Dürscheid, Christa. 2004. Netzsprache - ein neuer Mythos. Osnabrücker Beiträge zur Sprachtheorie (OBST) 68 (Thema des Heftes: Internetbasierte Kommunikation. Hgg. v. Michael Beißwenger; Ludger Hoffmann; Angelika Storrer), 141-157.

Dürscheid, Christa. 2016. Einführung in die Schriftlinguistik. Mit einem Kapitel zur Typographie von Jürgen Spitzmüller (UTB Sprachwissenschaften 3740). 5., aktual. und korr. Aufl. Göttingen: Vandenhoeck \& Ruprecht.

Dürscheid, Christa. 2018. Bild, Schrift, Unicode. In Guido Mensching et al. (Hgg.), Sprache Mensch - Maschine. Beiträge zu Sprache und Sprachwissenschaft, Computerlinguistik und Informationstechnologie. Köln: KUPS Kölner UniversitätsPublikationsServer, 269285. <https://kups.ub.uni-koeln.de/9849/> (6.12.2019).

Dürscheid, Christa; Frick, Karina. 2016. Schreiben digital. Wie das Internet unsere Alltagskommunikation verändert (Einsichten 3). Stuttgart: Alfred Kröner Verlag.

Dürscheid, Christa; Siever, Christina Margrit. 2017. Jenseits des Alphabets - Kommunikation mit Emojis. Zeitschrift für Germanistische Linguistik 45(2), 256-285.

Frick, Karina. 2018. Rezension zu: Veszelski, Ágnes. 2017. Digilect. The Impact of Infocommunication Technology on Language. Berlin \& Boston: de Gruyter. Zeitschrift für Rezensionen zur germanistischen Sprachwissenschaft. <https://doi.org/10.1515/zrs-2018-0024> (06.12.2019).

Gallmann, Peter. 1985. Graphische Elemente der geschriebenen Sprache. Grundlage für eine Reform der Orthographie (Reihe Germanistische Linguistik 60). Tübingen: Niemeyer.

Glück, Helmut; Rödel, Michael (Hgg.). 2016. Metzler Lexikon Sprache. 5., aktualisierte und überarbeitete Auflage. Stuttgart: Metzler.

Gredig, Andi. 2019. Die Spur der Gefühle. Kulturanalytische Überlegungen zum emotionalen Wert der Handschrift. In Stefan Hauser; Martin Luginbühl; Susanne Tienken (Hgg.), Mediale Emotionskulturen (Sprache in Kommunikation und Medien 12). Bern: Peter Lang, 3956.

Gunraj, Danielle N. et al. 2016. Texting insincerely. The role of the period in text messaging. Computers in Human Behavior 55, 1067-1075.

Klemm, Michael. 2016. Ich reise, also blogge ich. Wie Reiseberichte im Social Web zur multimodalen Echtzeit-Selbstdokumentation werden. In Kornelia Hahn; Alexander Schmidl (Hgg.), Websites \& Sightseeing. Tourismus in Medienkulturen. Wiesbaden: Springer VS, 31-62.

Krischke, Wolfgang. 2018. Mit Robotern reden. Steht die Linguistik vor dem großen Umbruch? Frankfurter Allgemeine Zeitung. Natur und Wissenschaft, N4, 10.1.2018.

Lobin, Henning. 2018. Digital und vernetzt. Das neue Bild der Sprache. Stuttgart: Metzler.

Lobin, Henning. 2014. Engelbarts Traum. Wie der Computer uns Lesen und Schreiben abnimmt. Frankfurt a.M.: Campus.

Nerius, Dieter et al. (Hgg.). 2007. Deutsche Orthographie. 4., neu bearbeitete Auflage. Hildesheim: Olms. 
Pappert, Steffen. 2017. Zu kommunikativen Funktionen von Emojis in der WhatsApp-Kommunikation. In Michael Beißwenger (Hg.), Empirische Erforschung internetbasierter Kommunikation (Reihe Empirische Linguistik 9). Berlin \& Boston: de Gruyter, 175-211.

Schubert, Christoph; Sanchez-Stockhammer, Christina (Hgg.). 2016. Variational Linguistics. Revisiting Register in English. Berlin \& Boston: de Gruyter.

Stöckl, Hartmut. 2004. Typographie: Gewand und Körper des Textes - Linguistische Überlegungen zu typographischer Gestaltung. Zeitschrift für Angewandte Linguistik 41, 5-48.

Storrer, Angelika. 2014. Sprachverfall durch internetbasierte Kommunikation? Linguistische Erklärungsansätze - empirische Befunde. In Albrecht Plewnia; Andreas Wit (Hgg.), Sprachverfall? Dynamik - Wandel - Variation (Jahrbuch des Instituts für Deutsche Sprache 2013). Berlin \& New York: de Gruyter, 171-196.

Storrer, Angelika. 2018. Interaktionsorientiertes Schreiben im Internet. In Arnulf Deppermann; Silke Reineke (Hgg.), Sprache im kommunikativen, interaktiven und kulturellen Kontext. Berlin \& Boston: de Gruyter, 219-244.

Ueberwasser, Simone; Stark, Elisabeth. 2017. What's up, Switzerland? A corpus-based research project in a multilingual country. Linguistik online 84(5), 105-126. <http://dx.doi.org/10.13092/lo.84.3849> (06.12.2019).

Veszelski, Ágnes. 2017. Digilect. The Impact of Infocommunication Technology on Language (Knowledge and Information/Studies in Information Science). Berlin \& Boston: de Gruyter. Zgraggen, Yvonne. 2017. „haha. die. pünkt. sind. voll. nötig.“ Untersuchungen zur Interpunktion in der WhatsApp-Kommunikation. BA-Arbeit Universität Zürich. <https://www.whats up-switzerland.ch/images/teaching/BA_Zgraggen.pdf> (06.12.2019). 
